\title{
The buckwheat iminosugar D-fagomine attenuates sucrose-induced steatosis and hypertension in rats
}

\author{
$\underline{\text { Sara Ramos-Romero }}^{1,2}$, Mercè Hereu $^{1}$, Lidia Atienza $^{3}$, Susana Amézqueta ${ }^{4}$, Josefina Casas ${ }^{5}$ and \\ Josep Lluís Torres ${ }^{1}$ \\ ${ }^{1}$ Institute of Advanced Chemistry of Catalonia (IQAC-CSIC), Barcelona, Spain, \\ ${ }^{2}$ Department of Cell Biology, Physiology \& Immunology, Faculty of Biology, University of Barcelona, Barcelona, \\ Spain, \\ ${ }^{3}$ Department of Pathology, Puerta del Mar University Hospital, Cádiz, Spain, \\ ${ }^{4}$ Departament d'Enginyeria Química i Quimica Analítica and Institut de Biomedicina (IBUB), Universitat de \\ Barcelona, Barcelona, Spain and \\ ${ }^{5}$ Research Unit on BioactiveMolecules (RUBAM), Department of Biological Chemistry, Institute of Advanced \\ Chemistry of Catalonia (IQAC-CSIC), Barcelona, Spain
}

\begin{abstract}
D-Fagomine (1,2 dideoxynojirimycin) is an iminosugar, a carbohydrate analogue that includes an endocyclic nitrogen instead of oxygen, that is naturally present in buckwheat and buckwheat-based foodstuffs. This study examines the long-term functional effect of d-fagomine on sucrose-induced factors of metabolic syndrome and explores possible molecular mechanisms behind its action. We evaluated Wistar Kyoto rats fed a standard diet were given a 35\% sucrose (glucose/fructose) solution with d-fagomine (or not, for comparison) or mineral water (controls) for 24 weeks. The variables measured were body weight and energy intake; glucose tolerance (oral glucose tolerance test); plasma leptin concentration; plasma lipid profile; the populations of Bacteroidetes, Firmicutes, bacteroidales, clostridiales, enterobacteriales, and Escherichia coli in feces; blood pressure; urine uric acid and $\mathrm{F}_{2 t}$ isoprostanes $\left(\mathrm{F}_{2}-\mathrm{IsoPs}\right)$; perigonadal fat deposition and hepatic histology and diacylglycerols (DAGs) in liver and adipose tissue. We found that d-Fagomine reduced sucrose induced hypertension, urine uric acid, $\mathrm{F}_{2}$-IsoPs as markers of oxidative stress (OS), steatosis and liver DAGs $(32: 1,32: 2,34: 1$ and 36:2) without affecting perigonadal (visceral) fat deposition or DAG levels in visceral adipose tissue. It showed a slight tendency to reduce sugar induced impaired glucose tolerance. d-Fagomine also promoted excretion of enterobacteriales generated by the dietary intervention. We postulate that fructose increases visceral fat deposition independently of liver de novo liposynthesis and that d-fagomine attenuates steatosis and blood pressure mainly by reducing liver fructose levels. The reduction of blood pressure may be associated with an effect on uric acid synthesis while the reduced levels of selected active liver DAGs may explain the weak effect on sucrose-induced impaired glucose tolerance, which may be primarily induced by visceral fat deposition. In conclusion, the increased populations of excreted enterobacteriales may be connected to the levels of excreted uric acid. d-Fagomine counteracts sucrose-induced steatosis and hypertension presumably by reducing the postprandial levels of fructose in the liver as a consequence of intestinal sucrase inhibition.
\end{abstract}

\section{Conflict of Interest}

There is no conflict of interest 